\title{
¿Coartación de aorta en el recién nacido? No siempre es lo que parece: a propósito de un caso
} Coarctation of aorta in a neonate? It is not always what it seems: an illustrative case

\author{
Dra. Alicia Martínez-Villasante Aleman ${ }^{a}$,Dr. Camilo López Socarrás ${ }^{b}$, Dra. Raquel Muñoz Muñizc, \\ Dr. Prof. Federico Gutiérrez-Larraya Aguado y Dra. Prof. Begoña Arias Novas ${ }^{a}$
}

\begin{abstract}
RESUMEN
La coartación de aorta en neonatos se puede manifestar como insuficiencia cardíaca según el grado de obstrucción. Hay situaciones que pueden simular una coartación de aorta en los recién nacidos. Limitarse a la imagen típica de muesca aórtica en la ecocardiografía para diagnosticar una coartación de aorta puede inducir a error y demorar el diagnóstico adecuado. Se presenta el caso de un recién nacido con insuficiencia cardíaca con diagnóstico inicial de coartación de aorta y, posteriormente, de malformación arteriovenosa cerebral. Se debe considerar la malformación arteriovenosa en el diagnóstico diferencial de un recién nacido con insuficiencia cardíaca.

Palabras clave: coartación aórtica, malformaciones arteriovenosas cerebrales, insuficiencia cardíaca.
\end{abstract}

\begin{abstract}
Coarctation of the aorta in neonates can manifest as heart failure when there is a certain degree of obstruction. There are some situations that can simulate a coarctation of the aorta in newborns. Diagnosis of coarctation of the aorta based solely on the typical aortic arch image on echocardiography can be misleading and delay an accurate diagnosis. We describe an unusual case of a newborn with heart failure who was initially diagnosed with coarctation of the aorta and then with cerebral arteriovenous malformation. We must consider the cerebral arteriovenous malformation in the differential diagnosis of a newborn with heart failure.
\end{abstract}

Key words: aortic coarctation, cerebral arteriovenous malformation, heart failure.

http: / / dx.doi.org/ 10.5546/ aap.2020.e67

Cómo citar: Martínez-Villasante Aleman A, López Socarrás C, Muñoz Muñiz R, Gutiérrez-Larraya Aguado F, Arias Novas B. ¿Coartación de aorta en el recién nacido? No siempre es lo que parece: a propósito de un caso. Arch Argent Pediatr 2020;118(1):e67-e71.

a. Servicio de Pediatría, Hospital Universitario Sanitas La Zarzuela, Madrid, España.

b. Servicio de Cardiología Pediátrica, Hospital Universitario Sanitas La Zarzuela, Madrid, España.

c. Unidad de Cuidados Intensivos Neonatales, Hospital Universitario Sanitas La Zarzuela, Madrid, España.

Correspondencia:

Dra. Alicia Martínez-Villasante Aleman: ali_mva@hotmail.com

Financiamiento: Ninguno.

Conflicto de intereses: Ninguno que declarar.

Recibido: 15-4-2019

Aceptado: 22-8-2019

\section{INTRODUCCIÓN}

La coartación de aorta (CoAo) es un estrechamiento congénito de la aorta, que presenta una prevalencia de 0,34 de cada 1000 recién nacidos vivos. ${ }^{1}$ Las manifestaciones clínicas varían según la edad de presentación. En los recién nacidos, se presenta como insuficiencia cardíaca; en los niños mayores, puede ser asintomática o inespecífica (intolerancia al ejercicio, epistaxis, cefalea). ${ }^{2}$ Se expone el caso de un recién nacido con insuficiencia cardíaca, con diagnóstico inicial de CoAo, cuyo curso clínico se complicó por hallazgos clínicos que dificultaron el correcto diagnóstico.

\section{CASO CLÍNICO}

Varón recién nacido tras un embarazo de curso normal, salvo por el hallazgo de regurgitación tricuspídea y cardiomegalia leve. Parto eutócico a las $38+3$ semanas, Apgar 9/10. Peso al nacer de 3200 g. Primer hijo de padres sanos, sin antecedentes de interés.

A las 20 horas de vida, ingresó en la Unidad de Cuidados Intensivos Neonatales del Hospital de origen por dificultad respiratoria. Exploración física: hipoactivo, tiraje subcostal e intercostal moderado, auscultación cardíaca con soplo sistólico de grado II/IV más audible en el foco tricuspídeo; el resto, normal. Se inició el soporte respiratorio (ventilación mecánica no invasiva). Radiografía de tórax con cardiomegalia global (índice cardiotorácico: $0,65)$ y congestión pulmonar (Figura 1). Fue evaluado por Cardiología Infantil con diagnóstico de hipertensión pulmonar persistente del recién nacido con presiones pulmonares suprasistémicas. Se inició el soporte inotrópico. Inicialmente, estable, presentó un empeoramiento progresivo (acidosis metabólica, oliguria y hepatomegalia, sin hipoxemia). Fue reevaluado por Cardiología y se destacó la presencia de insuficiencia tricuspídea grave, foramen oval permeable con shunt de derecha a izquierda, ductus grande bidireccional (predominio del shunt 
de derecha a izquierda) y arco aórtico con muesca posterior a la subclavia izquierda, sin doppler de coartación, con istmo de $5 \mathrm{~mm}$ (puntaje $\mathrm{Z}$ de $-0,77$ ) para aorta abdominal de 7,4 $\mathrm{mm}$ (puntaje $\mathrm{Z}$ de 1,64) y troncos supraaórticos dilatados. El paciente presentaba pulsos axilares y femorales normales, sin gradiente tensional. Se realizó una angio tomografía computada (angio-TC) y se observó la coartación de aorta torácica después de la salida de la arteria subclavia izquierda con cardiomegalia y dilatación de la arteria pulmonar.

\section{FIgURA 1. Radiografía de tórax}

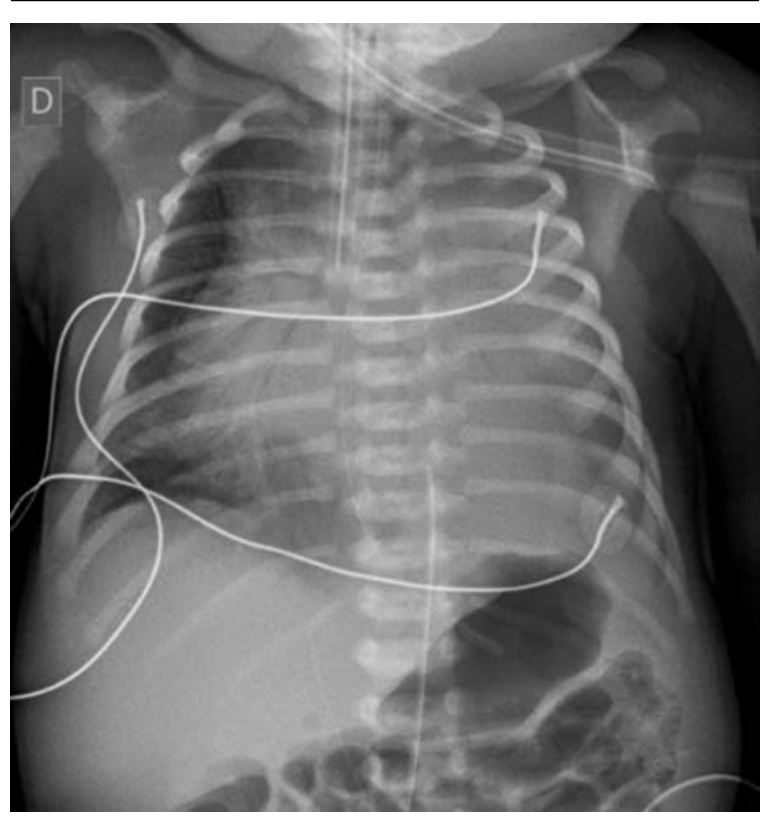

Cardiomegalia global y congestión pulmonar.
A las 72 horas de vida, fue trasladado a nuestro Centro. Presentaba frecuencia cardíaca de 180 latidos por minuto (lpm), tensión arterial de $50 / 35 \mathrm{mmHg}$ en los miembros superiores e inferiores, frecuencia respiratoria de 80 respiraciones por minuto $(\mathrm{rpm})$ y exploración física similar. Se inició la perfusión de prostaglandinas, que no toleró por desaturaciones graves y precisó la intubación orotraqueal. Se realizó una ecocardiografía y se observó gran dilatación de cavidades derechas, septo interauricular abombado a la izquierda con shunt de derecha a izquierda a través de foramen oval permeable, septo interventricular abombado a la izquierda, insuficiencia tricuspídea grave con gradiente sistólico máximo de $130 \mathrm{mmHg}$, hipertensión pulmonar grave, sin ductus; a nivel aórtico, no se apreciaba gradiente de coartación, pero se observaba una pequeña muesca en el arco aórtico posterior a la subclavia izquierda, y se apreció el robo diastólico en el arco aórtico (Figuras 2 y 3 ).

Con estos hallazgos clínicos y ecocardiográficos, se descartó el diagnóstico de coartación de aorta; por tanto, lo que se observó en la angio-TC fue la muesca del arco aórtico que simulaba una coartación.

Ante el cuadro de insuficiencia cardíaca derecha, hipertensión pulmonar grave y robo diastólico en el arco aórtico, se realizó una ecografía doppler cerebral y una resonancia magnética (RM) craneal por la sospecha de malformación arteriovenosa (MAV) cerebral, y se observaron fístulas arteriovenosas durales a lo largo del seno sagital superior y la tórcula

FIGURA 2. Ecocardiograma, plano supraesternal, eje largo

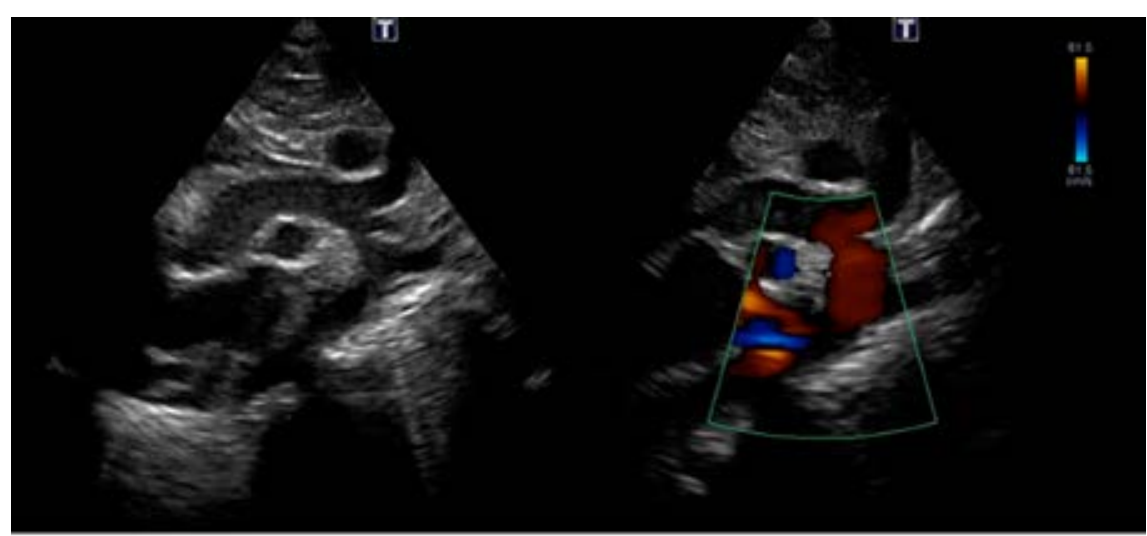

Arco aórtico con pequeña muesca posterior a la subclava izquierda y Doppler color con flujo diastólico retrógado (rojo)en arco aórtico. 
cuyas aferencias procedían de arterias meníngeas medias, arteria cerebral posterior derecha, arterias oftálmicas y ramas de las arterias occipitales. Se completó el estudio con angiografía cerebral, que confirmó el diagnóstico de MAV cerebral múltiple y se realizaron embolizaciones sucesivas de las fístulas arteriovenosas (Figura 4). Durante los procedimientos siguientes, presentó shock cardiogénico en relación con el infarto subendocárdico y taquicardia auricular con repercusión hemodinámica, que precisó cardioversión eléctrica y recuperó el ritmo
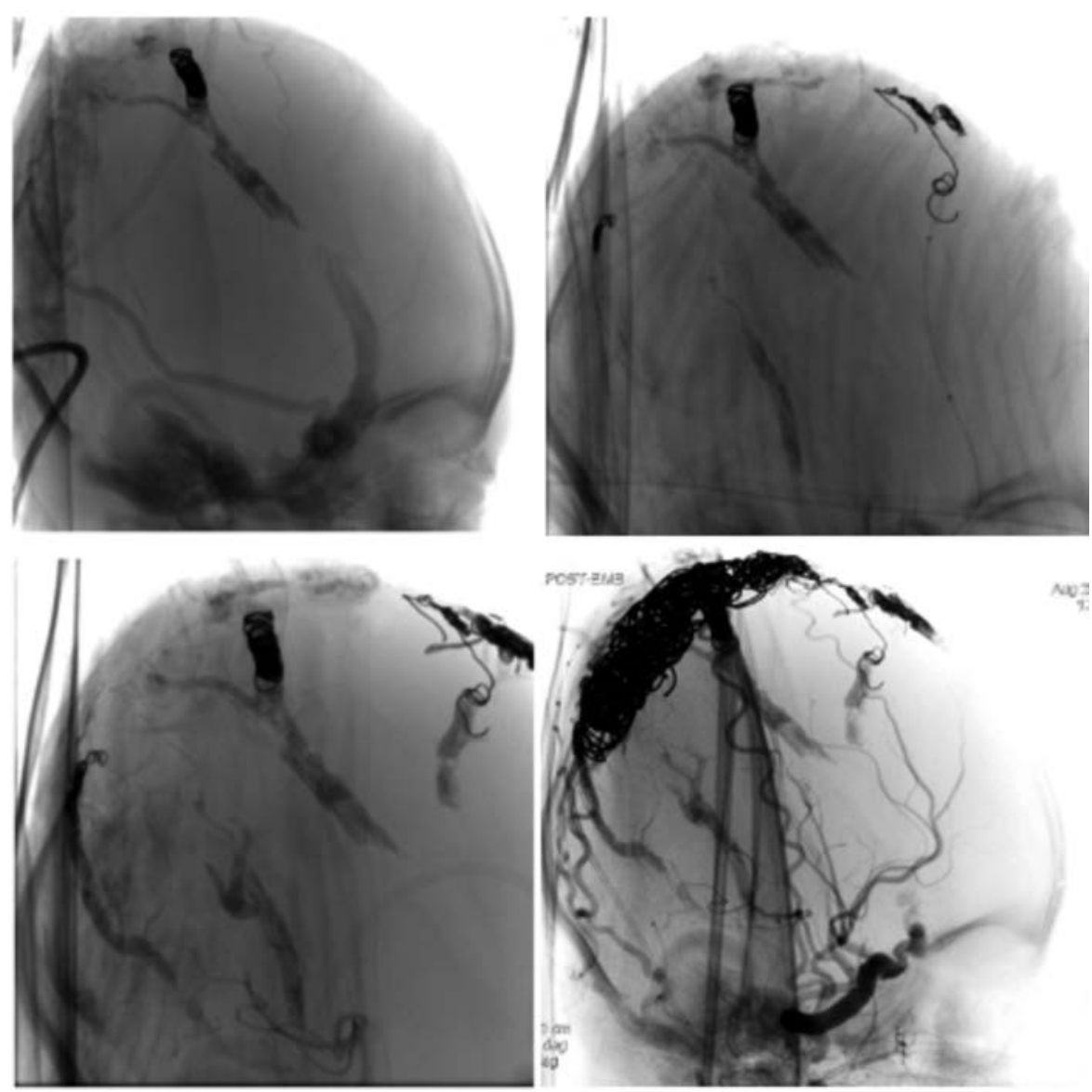

Embolizaciones sucesivas de las fístulas arteriovenosas.

FIGURA 3. Ecocardiograma, plano apical de cuatro cámaras

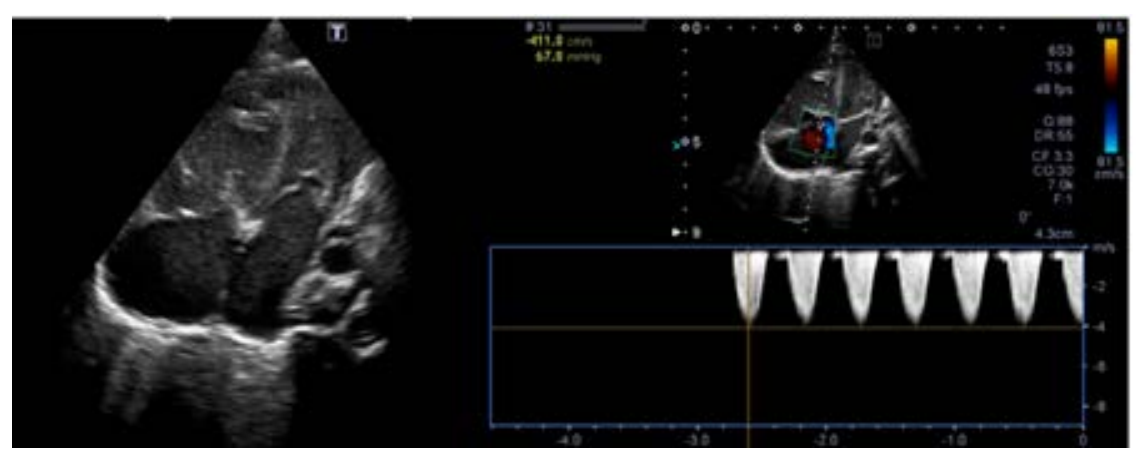

Dilatación de cavidades derechas y septo interventricular abombado a la izquierda. Doppler continuo en válvula tricúspide donde se observa insuficiencia tricuspídea. 
sinusal. Requirió cuatro embolizaciones hasta controlar la insuficiencia cardíaca. Se realizó una RM cerebral tras las embolizaciones y presentó menor dilatación de los senos venosos durales intracraneales e hidrocefalia secundaria sin deterioro neurológico, por lo que se decidió una actitud expectante con controles ecográficos seriados y se observó una disminución progresiva de la hidrocefalia hasta su resolución. Al momento del alta, la exploración era normal. Actualmente, tiene 12 meses y recibe seguimiento en Cardiología Infantil sin precisar tratamiento farmacológico y en Neuropediatría con desarrollo psicomotor normal.

\section{DISCUSIÓN}

La CoAo consiste en una obstrucción al flujo sanguíneo, generalmente, en la parte superior de la aorta descendente a nivel del ductus. La gravedad de la estenosis va a determinar los cambios hemodinámicos que se produzcan en el recién nacido. La CoAo en recién nacidos se manifiesta como insuficiencia cardíaca tras empezar la constricción del ductus. Es característica la diferencia de pulsos y de presión arterial sistólica entre las extremidades superiores e inferiores, con pulsos débiles por debajo de la CoAo, presión arterial aumentada en las extremidades superiores y un gradiente de presión mayor de $20 \mathrm{mmHg}$ entre los miembros superiores e inferiores. Se puede auscultar un soplo sistólico eyectivo en el borde esternal izquierdo irradiado a la región interescapular izquierda. La ecocardiografía doppler es el método más utilizado para el diagnóstico, y se visualiza el estrechamiento de la aorta con aceleración del flujo, aumento del gradiente sistólico pico, prolongación diastólica, hipoplasia del istmo, dilatación posestenótica y disminución del flujo tras la coartación. ${ }^{3}$

Hay algunas afecciones que pueden simular una CoAo. En una ecocardiografía que muestre el aumento de cavidades derechas, ductus arterioso persistente y flujo diastólico retrógrado en cayado aórtico, en ausencia de disfunción sistólica grave del ventrículo izquierdo, se debe buscar de forma activa una derivación izquierda-derecha, como una fístula aortopulmonar, una MAV cerebral o cualquier fuente de robo diastólico., ${ }^{4,5}$

Las MAV cerebrales son errores de la morfogénesis vascular que surgen de la persistencia de conexiones directas entre la arteria y la vena embrionarias por falta de desarrollo de la red capilar interpuesta. ${ }^{6}$ En los neonatos, las manifestaciones más frecuentes son insuficiencia cardíaca, macrocefalia y, al momento de la exploración, la auscultación de soplo intracraneal. ${ }^{7,8}$

Las MAV intracraneales que conducen a la insuficiencia cardíaca en el recién nacido fueron descritas por primeras vez por Gómez et al. en $1963 .{ }^{9}$ Las MAV cerebrales proporcionan una derivación arteriovenosa que provoca un flujo sanguíneo cerebral aumentado y acelerado debido a la disminución de la resistencia vascular de la MAV; por tanto, una cantidad desproporcionada del flujo sistémico se va a desviar hacia la MAV. ${ }^{6}$ Una MAV cerebral en un recién nacido puede simular una CoAo al manifestarse como insuficiencia cardíaca. ${ }^{7}$ Una teoría propuesta para explicar la imagen de la CoAo en una MAV cerebral sostiene que el aumento del flujo de salida del ventrículo derecho en el feto conduce a un aumento del flujo a través del ductus arterioso y un flujo anterógrado reducido a través del istmo aórtico. La baja resistencia en la MAV cerebral conducirá a una disminución del flujo anterógrado desde la aorta ascendente hasta el istmo. La imagen de coartación de aorta se produce porque el istmo es el segmento más estrecho del arco aórtico, mientras que la aorta ascendente y transversa estarán dilatadas por el aumento del flujo hacia la MAV cerebral. ${ }^{10}$

Los hallazgos ecocardiográficos en las MAV cerebrales son dilatación de la vena cava superior con dilatación de cavidades derechas debida a un aumento de la precarga cardíaca, flujo diastólico retrógrado en la aorta descendente y/ o cayado aórtico, y un aumento de las velocidades de flujo en todas las válvulas cardíacas y grandes vasos debido al alto gasto cardíaco. ${ }^{11}$

En la RM cerebral, se puede detallar la MAV cerebral, pero el gold standard para el diagnóstico es la angiografía, que permite identificar los vasos implicados y los detalles anatómicos de la malformación. ${ }^{12}$

El tratamiento de elección es la embolización endovascular mediante neurorradiología intervencionista. El tratamiento ha aumentado la supervivencia, aunque aún se está intentando estimar el pronóstico neurológico de estos pacientes. ${ }^{13} \mathrm{El}$ pronóstico en la insuficiencia cardíaca secundaria a MAV cerebral durante el período neonatal es malo. En la serie de García-Mónaco et al. ${ }^{14}$ revisaron el manejo de 30 niños con insuficiencia cardíaca por MAV cerebral y obtuvieron una mortalidad del $20 \% .{ }^{14}$ Es importante un manejo agresivo de la 
insuficiencia cardíaca antes de la embolización y un tratamiento precoz intervencionista para disminuir la morbimortalidad y evitar secuelas en el desarrollo neurológico. ${ }^{15}$

En conclusión, hay situaciones que pueden simular una CoAo, como muestra este caso. Ante un recién nacido con insuficiencia cardíaca, es fundamental evaluar al paciente en su conjunto realizando una exploración física detallada (que incluya la auscultación de la fontanela anterior) unida a la información que aportan las pruebas complementarias para poder realizar un diagnóstico y tratamiento correctos. Se debe sospechar MAV cerebral en todo recién nacido con cardiomegalia e insuficiencia cardíaca en ausencia de una cardiopatía estructural que presente una ecocardiografía doppler con flujo retrógrado en el arco aórtico.

\section{REFERENCIAS}

1. Van der Linde D, Konings E, Slager MA, Witsenburg M, et al. Birth prevalence of congenital heart disease worldwide a systematic review and meta-analysis. J Am Coll Cardiol. 2011; 58(21):2241-7.

2. Luca AC, Horhota OE. Clinical and epidemiological aspects in coarctation of the aorta in children. SEA. 2017; 5(15):483-6.

3. Centella Hernández T, Stanescu D, Stanescu S. Coartación aórtica, interrupción del arco aórtico. Cir Cardiovasc. 2014; 21(2):97-106.

4. Bagdure D, Bartakian S, Kaufman J. Coarctation of aorta and vein of Galen aneurysmal malformation in a neonate. Curr Opin Pediatr. 2011; 23(2):249-52.
5. Harahsheh AE, Kulkarni A, Becker C, Ross RD. Conditions mimicking coarctation of the aorta. Pediatr Cardiol. 2007; 28(5):385-8

6. Baskaya MK, Jea A, Heros RC, Javahary R, et al. Cerebral arteriovenous malformations. Clin Neurosurg. 2006; 53:114-44.

7. Herman TE, Siegel MJ. Congenital dural arteriovenous fistula at torcula Herophili. J Perinatol. 2007; 27(11):730-1.

8. Roccatagliata L, Bracard S, Holmin S, Soderman M, et al. Pediatric intracranial arteriovenous shunts: a global overview. Childs Nerv Syst. 2013; 29(6):907-19.

9. Gomez MR, Whitten CF, Nolke A, Bernstein J, et al. Aneurysmal malformation of the great vein of Galen causing heart failure in early infancy: report of five cases. Pediatrics. 1963; 31(3):400-11.

10. McElhinney DB, Halbach W, Silverman NH, Dowd CF, et al. Congenital cardiac anomalies with vein of Galen malformations in infants. Arch Dis Child. 1998; 78(6):548-51.

11. Doyle NM, MastrobattistaJM, ThaparMK, Lantin-Hermoso MR. Perinatal pseudocoarctation echocardiographic findings in vein of Galen malformation. J Ultrasound Med. 2005; 24(1):93-8.

12. Yu J, Lv X, Li Y, Wu Z. Therapeutic progress in pediatric intracranial dural arteriovenous shunts: A review. Interv Neuroradiol. 2016; 22(5):548-56.

13. De Rosa G, De Carolis MP, Tempera A, Pedicelli A, et al. Outcome of neonates with vein of Galen malformation presenting with severe heart failure: a case series. $A m \mathrm{~J}$ Perinatol. 2019; 36(2):169-75.

14. García-Mónaco R, De Víctor D, Mann C, Hannedouche A, etal. Congestive cardiac manifestations from cerebrocranial arteriovenous shunts. Endovascular management in 30 children. Childs Nerv Syst. 1991; 7(1):48-52.

15. Frawley GP, Dargaville PA, Mitchell PJ, Tress BM, et al. Clinical course and medical management of neonates with severe cardiac failure related to vein of Galen malformation. Arch Dis Child Fetal Neonatal Ed. 2002; 87(2):144-9. 